\title{
History of Medicine and Advanced Manufacturing for Modernization of Health Services: The New Paradigm for the Learning and Practicing of Medicine Shortly Roberto Badaró*, Josiane Dantas Viana Barbosa and Leone Peter Andrade \\ Deputy Editor, Associate Editor and Editor-in-Chief
}

\section{History Perspectives}

The term advanced manufacturing is closely linked to industrialization. However, the classical definition in the Manufacturing dictionaries is a production system where the production technique, despite being handcrafted, organizes the division of labor in a more complex and reproductive way that is not identified in the craftsmanship. The history of teaching is vibrant and millenarian dating from the Neolithic period 6000 BC. Today, it is a skill capable of transforming nature with its best practices and remains a practice of all civilizations. However, industrial-scale production is a consequence of manufacturing learning by transferring what is done with the hands to be performed by the machines. Therefore, advanced manufacturing is transferred to make the things with hands to make with the tools; it's a machine replacing doing it with your hands. The enormous impact caused by advanced manufacturing appeared with a industrial revolution after World War II.

Human-made devices produce consumption goods, and soon after that the concept of automation diminished or even eliminated in many sectors of the productive activity of man participation. From the didactic point of view, there are numerous publications of books and texts that classify the evolution of the manufacture in revolutionary marks, textually designating its development in the Industrial Revolution.

The first revolution, assigned Industry 1.0, characterized by mechanization in the production of consumer goods occurred in the 2nd Industrial Revolution in 1945. It was the beginning of the era of the explosion engines. The generation of energy by combustion modernized the craft, created the actual manufacture. In the $1960 \mathrm{~s}$, the combination of electronics and mechanization began the 3 rd Industrial

Address for correspondence: Roberto Badaró. Avenida Orlando Gomes, 1845, Piatã.Zip Code: 41650-010, Salvador-Bahia-Brazil. Phone: (55 71)3879-5501; e-mail: badaro@fieb.org.br.
Revolution. The mass production of consumer goods and the introduction of TV and radio started new manufacture to Industry 2.0. The possibilities of controlling industrialization through software and computers, as well as the domain of electronic transmission over long distances with optical fibers and electromagnetic waves, amplified the power of communication with intercontinental limits and opened the way for the construction of robotics, making possible the automation in manufacturing. Thus, the beginning of Industry 3.0. In the 1980s, the acceleration of advanced building with computers and mastery of robotics revolutionized the way of doing. Discovering wireless communication by Bluetooth, $\mathrm{Wi}-\mathrm{Fi}$, internet and digital control transferred the execution of things by the connection. The smartphones that can operate remote machines almost eliminate the strength of the human arms in the productive industry process. Thus, this inaugurated the beginning of Industry 4.0.

Summary of the Advanced Manufacturing Markings years:

1945 Industrial Revolution (mechanization in the production of consumer goods);

1960 Electronic Revolution (introduction of TV, radio);

1970 Communication Revolution (intercontinental transmission);

1980 Computer Revolution;

1990 Revolution of Cybernetics (internet);

2000 Digital Revolution: smartphones, IOT, artificial intelligence, robotics.

\section{The Modernization of Health by the Influence of Advanced Manufacture}

The Hippocratic medicine ends with the end of the handcraft in the production of surgical instruments and of medical assistance in the practice of medicine. The oldest medical device is the knife. The surgical blade dates back to the Mesolithic period, around $8000 \mathrm{BC}$. One of the earliest surgical procedures was trepanation, the art of piercing a hole in the skull. The results suggest 
that flint knives were created to perform the perforation of the head was opened to allow the release of the demons that caused headaches, melancholy or other epilepsy to escape the body. In the middle ages, practically all Aesculapius's dedicated to medical art had their suitcase, however, huge jumps have occurred in the evolution of medicine, and more and more intervals are being smaller in the modernization of medicine.

At the Stone Age 3300 years BC, in the so-called Medieval Medicine, the concept of helping sick people was through the administration of potent herbs that served as food but cause some effects on the body, and sometimes they recovered people from their evils. The other great landmark of medicine happened 420 BC. Hippocrates, considered the father of medicine, writes the code of ethics that doctors should follow in the face of the brutality that Aesculapius performed to cure illness.

The Hippocrates era goes up to 200 AD. Galen, $200 \mathrm{AD}$, influenced by the writings of illustrious physicians at the time and the knowledge of Cornelius Celsus (25BC-50 AD), wrote the first encyclopedia of medicine, beginning the era of medicine based on observation and experience. The dose of the drug makes the poison. Julian, the apostle, who was Roman emperor from 361-363 AD, in imitation of Christian charity to care for the sick inaugurates, in imitation of Christ, the place of caring for the sick, the hospital. In the year 369 AD, Basil Caesaria, bishop of Caesaria Masaca in Cappadocia, consolidates the concept of a hospital with several buildings, already with specialties, doctors, nurses, workers, and medical teaching place, creating a real hospital separating patients from their residence.

Ephren of Syria in the year 375 AD in Mesopotamia, consolidates the hospital by separating the types of diseases in different hospitals for different conditions and even creates a place of lodging and housing for the orphans. In 420 AD, Caelius Arelianus, a Roman physician writes the encyclopedias of medicine dividing diseases into acute and chronic. It is the beginning of specialization in medicine. Until the year $1161 \mathrm{AD}$, medicine evolved in a breakaway attempt at surgical diseases and clinical diseases. The church with Pope Innocent III from 1203 to 1493 AD had an enormous influence on the evolution of specialization in medicine. Vocational as a Christian activity the care of the sick and creates hospitals throughout Europe and colonies of the Roman Empire were fast developed. High schools and invention in medicine arise. The anatomy allows the knowledge of the human body, the design of the lens returns the vision in myopia and farsightedness. In the period 1307-1392 $\mathrm{AD}$, surgery is considered non-palliative but curative with the surgical techniques described by the English surgeon, John Ardene, considered the father of the operation. Many of his techniques are still employed today. In the year $1500 \mathrm{AD}$, Paracelsus, an alchemist who knows metals and minerals, inaugurated a new paradigm in medicine with the use of chemical substances in medical treatment. In 1543, Andreas Vesalius deepened the knowledge of anatomy, and the drug became a Cartesian activity definitely. The same wisdom of Descartes, in his discourse on method, became used in the study of the human body allowed to describe the organs and human body systems. However, the great invention that accelerates the discovery of diseases and their association with an etiology was in the period 1500 to $1600 \mathrm{AD}$ with the development of the microscope. Although Zacharias Janssen was recognized in 1590, as the inventor of the lens, his real discoverer, 50 years before, is unknown. In the period from 1601-1900 many significant discoveries in medicine stems from the use of the microscope. Discovery of cells and microorganisms all occurred in this period. Some events are listed below:

1518 The foundation of the first college of surgeons training - the Royal College Physicians of London;

1774 Joseph Priestley discovered nitric oxide, ammonia, hydrogen chloride, and oxygen;

1775 Samuel Hahnemann discovered homeopathy;

1776 Edward Jenner developed the vaccine against smallpox;

1799 Hamphry discovered the anesthetic properties of nitrous oxide;

1816 Rene Laennec invented the stethoscope;

1818 James Blundell performed a first blood transfusion;

1827 Joseph Lister started the antisepsis applied to the new surgery; 
1842 Crawford Long performed the first surgery using ethyl ether in anesthesia;

1845 John Hughes Benett described leukemia and blood diseases;

1846 Starts painless operations with the use of general anesthesia;

1847 Ignaz Semmelweis created the concept of hospital infection with the association of puerperal fever with the contamination of the hands in obstetrician in the deliveries;

1849 Elizabeth Blackwell was the first woman trained in medicine in the United States;

1858 Rudolf Carl Virchow created the concept of the pathology associated with cellular alterations combating the idea of the pathology of humor;

1867 Lister published the principles of asepsis in the performance of surgeries based on the studies of Louis Pasteur;

1870 Louis Pasteur and Robert Koch created the theory of diseases caused by germs;

1879 The invention of the cholera vaccine;

1881 Louis Pasteur developed the pest vaccine;

1882 Louis Pasteur developed the anti-rabies vaccine;

1890 Emil von Behring discovered the anti-toxins and used against tetanus and diphtheria.

\section{Electricity and Magnetism Boost New Diagnostic Methods}

Breakthrough technologies began to be discovered.

1895 Wilhelm Conrad Rontgen discovered the X-ray and used it to realize the radiographs of the human body;

1910 Hans Jacobaeus performed the first laparoscopy on humans;

1921 Frederick Banting and Charles Best discovered insulin - essential for the treatment of diabetes;

1928 Alexander Fleming discovered the penicillin;

1929 Hans Berger discovered the electroencephalography;

1946 Alfred Gilman and Louis Goodman used the chemotherapy;

1949 Harold Ridley performed the first implant intraocular; John Emerson created a mechanical assistor for anesthesia;

1959 Min Chueh Chang used the in vitro fertilization;

1960 Invention of cardiopulmonary resuscitation (PCR);

1962 Albert Sabin discovered the first oral polio vaccine (Sabin);

1963 Thomas Starzl performed the first human transplant;

1965 Frank Pantridge installed the first portable defibrillator;

1967 Christiaan Barnard performed the first human heart transplant.

\section{Computing and Software Revolutionize} Diagnostic Imaging Methods

1971 Raymond Vahan Damadian created the magnetic resonance imaging (MRI);“ Godfrey Hounsfield discovered the computed tomography (CT or CAT Scan);

1972 Mani Lal Bhaumik performed the first laser eye surgery (LASIK);

1973 Giorgio Fischer create the liposuction;

1985 Leroy Hood and Lloyd Smith discovered the automated DNA sequencer;

Kary Mullis discovered the polymerase chain reaction.

\section{Molecular Biology and Genome Discovery Along with Robotics}

Begins the Era of Automation in Medicine

1985 Yik San Kwoh began the surgical robotic;

1988 Julio Palmaz used the first intravascular stent;

1996 Dolly, the sheep, was the first animal cloned;

1998 James Thomson started to use the steam cell therapy;

2000 The Human Genome Project draft was completed;

2013 The first Kidney has grown in vitro in the U.S.;

The first Human liver has grown from stem cells in Japan;

2014 The first 3D was used for first ever skull transplant;

2016 The first ever artificial pancreas was created.

\section{Significant Advances that are Modifying Medicine in the 21st Century}

Each year, when we look at Google and other information research sites on the internet about what is happening in the world of medical science, the list of necessary technologies increases dramatically. Within today, the medical curriculum has lagged, bringing a fundamental issue to universities that graduate doctors and health professionals. Are we training health professionals able to care for patients in the light of the most appropriate scientific knowledge to be used in medical practice? Even with the post-graduation incentive and the professional strategies of the division of medical expertise in subspecialties, we still cannot incorporate innovation simultaneously into the practice of medicine. Besides, changes in the demographic profile of populations around the world, life expectancy and the nosological profile of diseases associated with aging make medical and hospital institutions that care for and prevent illnesses, obsolete. The forecast for demographic and socio-economic evaluation institutes 
in large parts of the world is that by 2050 we will have a population of 9 billion people in the world, and 400 million people in Brazil, what will force us to dramatic changes in health systems. The European example reveals a decrease in the number of hospital beds. Thus, the new paradigm for the provision of health services was modernized to preferentially outpatient care. Using virtual medical offices, shared clinics, home care of patients and active participation of the internet in the provision of consulting and services in the health area, advances in diagnostic and therapeutic methods in medicine are considerable increasingly targeting health services for individualized therapy.

There will be no more indiscriminate chemical action medications. The therapeutic approach will increasingly be through individualized protocols using individualized genomic therapy targeted to the genome of each person. Specific treatment for groups of diseases and their particular manifestations of each peculiar condition will propel a trend towards virtualization and a transition from manufacturing, to individualized drug treatment centers. Pharmaceutical companies are increasingly aiming to provide holistic services, including algorithmdriven data processing, case management, and billing guidance.

However, this should not be confused with the business models of health maintenance organizations (HMOs) that exist in the United States, which already migrated to Brazil. There is a paradigm shift in the health industry driven by new technologies and strategies. One such approach is Industry 4.0, which is not restricted to the consumer goods manufacturing industries but seems to fit the health domain well. The Hospital 4.0 concept is an extension of the Industry 4.0 concept, meaning the fourth industrial revolution that includes cyber-physical systems, the Internet of Things (IoT) and cloud computing and artificial intelligence. Through the Internet of Things, cyber-physical systems communicate and cooperate with each other and with humans in real time, and through the Internet of the Services. These aspects are increasingly becoming a reality in modern hospitals that are generally up to date with technology, which emphasizes the Hospital 4.0 concept more strongly than the Industry 4.0 concept.
These aspects are increasingly, and as consequence, healthcare will be much more related to interoperability: the ability of equipment, devices, sensors, and people to connect and communicate through the Internet of Things (IoT) or the Internet of People (IoP); in modern hospitals, the monitors and procedures for the execution of a medical service are trending to be individualized; nursing stations are frequently being transferred to automated computer-operated systems directly from the patient's room.

There are four design principles in Hospital 4.0 concept:

a) Virtual Hospital - The construction of hospitals will be through information systems that create a virtual image of real hospital models through data sensors;

b) Maintenance - The ability to support systems availability and help people maintain them, including the use of newer tools such as Augmented Reality and Holography.

c) Decentralized Decisions - The ability to perform tasks at each level through cybernetic systems in the most independent way possible.

d) $\mathbf{R} \& \mathbf{D}$ Activities - These systems applied to the hospital reality increasingly incorporate R \& D activities in Health Engineering; Asset Management; Risk management; Infections associated with healthcare, Certifications; Energy; Sustainability and Multidisciplinary Engineering becoming a reality in modern hospitals that are generally up to date with technology, which emphasizes the Hospital 4.0 concept more strongly.

At the last Brazilian Industrial Association of Medical and Dental Articles and Equipments (ABIMO) meeting, the ideas of Industry 4.0 for the hospital system were discussed, concluding that those above principals are an extension of the original concept.

\section{Main Technologies that are Being Applied to Healthcare}

After Hippocrates (460 BC), the great innovation in medicine had happened a thousand years later. The invention of the magnifying glass to enable man to see what Galileo with his telescope perfected to understand the universe, led Robert Hooke to create the microscope to see the invisible, as well as Benjamin Franklin's electricity with the control of lightning electricity, and Thomas Edson and the dazzling light that led to Roentgen discovered the X-ray, Godfrey 
Hounsfield, and Raymond Vahan Damadian made the magnetism and computer originating the computed tomography and the magnetic resonance. Now the new machines are of the $3 \mathrm{D}$ reality of electronically captured images. Indeed, modern medicine will be developed on the platform of 10 major technology fields:

1. $3 \mathrm{D}$ printers;

2. The boom of medical applications: CRISPR Genome Editing (curing disease at a genetic level); electronic skin that can read body's vital signs, cancer vaccines, brain implants, transplant polymers, ingestible sensors;

3. Telemedicine;
4. Uber model in health;

5. Nanomedicine;

6. Robotic surgery;

7. Virtual reality;

8. Food scanners;

9. Fibertronics;

10. Artificial intelligence.

The creation of JBTH now is a great opportunity for young investigators that are looking for good journals to publish a scientific paper and innovations fast and with an interface with engineering and medicine that it is not appropriated covered in the existing journals. 